\title{
Ultrasound Imaging with Microbubbles
}

\author{
Antonio Stanziola, Matthieu Toulemonde, Yesna O. Yildiz, Robert J. Eckersley, Meng-Xing
}

Tang

\section{Introduction}

Medical ultrasound (US) imaging, also known as echography, is one of the most frequently used front-line clinical imaging modalities and is characterised by its safety, affordability, accessibility, and real-time image display. Sound pulses, typically in the mega-hertz range, are sent into the body and the backscattered echoes are used to create a tomographic image. The contrast of an US image arises from local variations in the physical properties of the tissues, primarily density and elasticity, revealing tissue structures at depth.

The blood flow of a living organism contains essential information related to tissue function and pathology. However, as blood is a fluid composed of plasma and blood cells which are similar to each other acoustically the scattering of sound from blood is very weak compared to surrounding tissue structures. Consequently it is difficult to detect vessels which are smaller in size than the pulse length, as the echoes from blood are completely masked by the neighbouring tissue response. While for big vessels it is still possible to obtain hemodynamic information due to the weak scattering of blood cells, in general, the signal to noise is poor and the information content is limited.

To overcome the limitations of conventional US, gas bubbles of micrometer radius can be introduced into a patient's blood stream as agents for contrast enhanced ultrasound imaging (CEUS). Microbubbles act as resonant oscillators and scatter ultrasound signals efficiently when excited at the frequencies used in the clinical practice, typically in the range of $1-15 \mathrm{MHz}$. They are designed to have a diameter ( $<7$ microns) capable of passing the pulmonary capillaries (the smallest vessels in the human body) and often include a lipid shell which encapsulates a low solubility gas, so as to increase their longevity in the circulation.

The use of microbubbles (MBs) in US imaging generates new exciting possibilities [1]. It enables real-time imaging of blood flow with unprecedented sensitivity and resolution in both large vessels and microvasculature, and provides indicators of the perfusion of organs in e.g. liver (Fig. 1(a-b)), kidney (Fig. 1(c-d)), heart (Fig. 4(a)), limbs, brain, breast and lymphatic systems. The safety of MBs in diagnostic ultrasound has been well established. If the MB shell is coated with specific molecules, complementary to those expressed by the vascular walls during specific pathological processes such as the initiation of cancer or atherosclerosis, it is 
possible to bind $\mathrm{MBs}$ to these receptors facilitating molecular imaging for early detection and diagnosis $[1]$.

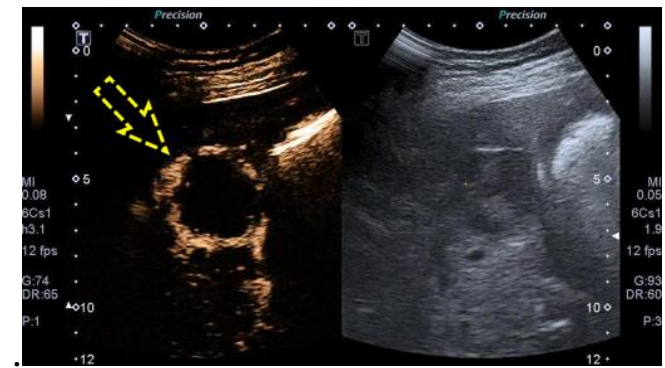

(a)

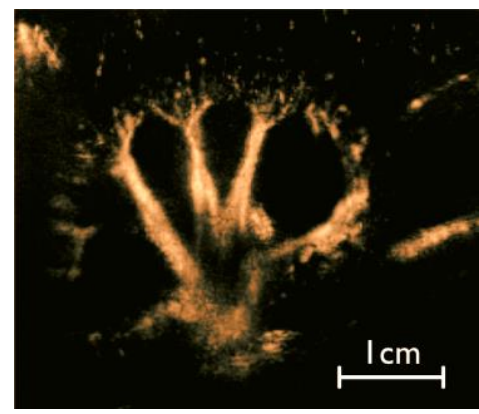

(c)

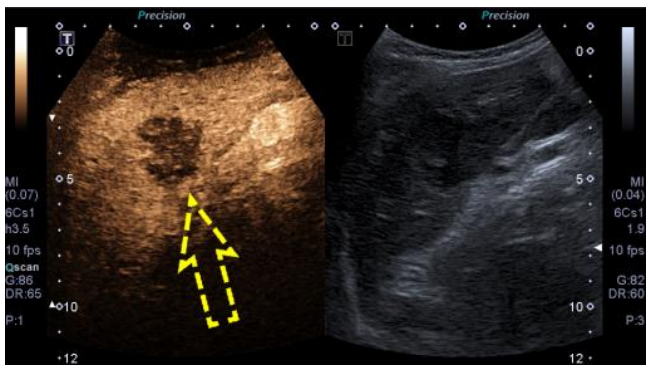

(b)

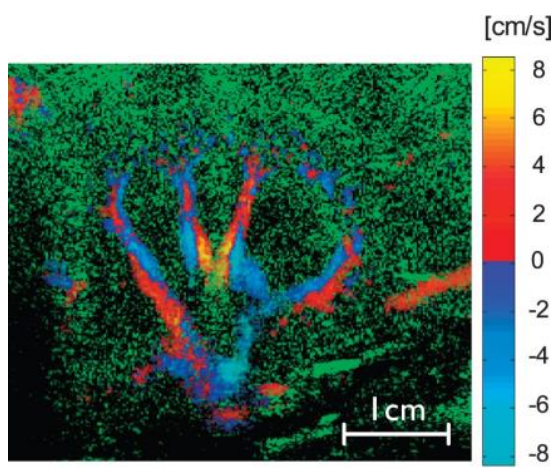

(d)

Figure 1: Top: comparison of side by side CEUS contrast specific image (colour) vs. standard US B-mode image (gray scale) for a liver arterial haemangioma (a) and a liver metastasis (b). Both lesions are clearly visible in the contrast specific images but not the standard B-mode images. In the contrast specific images the haemangioma is shown to be surrounded by a ring of contrast enhancement (arrow in (a)), while the metastasis is shown as a dark area (arrow in $(b))$.

Bottom: CEUS image (c) and nonlinear Doppler CEUS image (d) of a rabbit kidney vasculature (adapted from [2]). Nonlinear Doppler shows additional information including the direction of flow (blue and red) as well as microcirculation information (green).

\section{Microbubbles as Nonlinear Oscillation Systems}

The equilibrium radius of a $\mathrm{MB}$ is a balancing act between the external pressure of the blood, the internal pressure of the gaseous core, the shell elastic properties, and the surface tension. When a US wave excites an MB, since the bubble diameter $\left(\sim 10^{-6} \mathrm{~m}\right)$ is much smaller than the clinical US wavelength $\left(\sim 10^{-4} \mathrm{~m}\right)$, it causes a global change in the external pressure $p_{0}$ that results in an oscillation of the MB, associated with the emission of sound waves (Fig 2(a-b)). As the gas core is highly compressible, the bubble radius can oscillate significantly 
around its equilibrium value $r_{0}$ if driven at or near its resonance frequency, and this behaviour is responsible for the strong scattering of the contrast agents.

MBs are inherently nonlinear oscillation systems. Even with US amplitudes of tens of kilopascal, well below those typically utilized in clinical imaging, the radius change for an MB is not symmetrical in the compression and expansion phases. This asymmetry is a key source of harmonic generation from MBs.

The MB oscillation and subsequent scattering is highly dependent on the US parameters, including frequency and amplitude. A variety of analytical models have been developed to describe this process, most of them assuming a time-invariant spherical bubble. The simplest is the Rayleigh-Plesset (RP) model, which considers a shell-free bubble of polytropic gas in an inviscid incompressible fluid of density $\rho$. If $p_{\infty}$ is the liquid pressure far away from the bubble and $p=p(t)$ the pressure at the bubble interface, the time evolution of the radius $r$ is governed by:

$$
\ddot{r} r+\frac{3}{2} \dot{r}^{2}=\frac{p-p_{\infty}}{\rho}
$$

where $\dot{r}$ and $\ddot{r}$ are respectively the first and second derivatives of $r(t)$ with respect to time. The non-linear term on the left side shows that bubble oscillation in response to a pressure wave is non-linear and, for a sinusoidal excitation, the scattered signal will contain harmonics and sub-harmonics (Fig. 2(c)). This has been verified experimentally and, since the scattering from existing structures in the body is generally considered to be linear, this is the main characteristic exploited to distinguish between MBs and tissue signals.

The nonlinear behaviour of a MB can be further enhanced by the presence of a shell. The RP equation has therefore been refined to include the effects of the shell, the surface tension at the bubble interface, the viscosity of blood, and the finite speed of sound, leading to a number of modified Rayleigh models [3].

\section{Detecting Nonlinear Scattering Signals from Microbubbles}

In order to image the MBs in small vessels, the tissue signals need to be removed to facilitate generation of MB specific images. While it is possible to use a high-pass filter, with a cut-off frequency between the fundamental and second-harmonic $(\mathrm{SH})$ frequencies, to remove the signals reflected by tissues, this approach is fundamentally limited by the transducer bandwidth. In US imaging, a short broadband pulse is required as the spatial resolution is determined by the transmitted pulse length. It is difficult to completely separate the broadband fundamental signal from its harmonics within a limited bandwidth, reducing the contrast 
between MBs and tissue (Fig. 2(h)).

\subsection{Multi-pulse acquisition}

Multi-pulse techniques such as Pulse Inversion (PI) have been developed to elegantly separate the MB signals from tissue without sacrificing spatial resolution. In PI, two identical but phase-inverted pulses, as shown in Fig. 2(d), are transmitted into the media consecutively. While either echo of the two pulses can produce a conventional US image (B-Mode image) (Fig. $2(\mathrm{~g}))$, the sum of the two echoes is zero for linear scatterers (tissue) while for nonlinear scatterers (microbubble) the sum is non-zero, see Fig. 2(e). In the frequency domain shown in Fig. 2(f), for PI, all even harmonics are preserved while the fundamental signal $(3 \mathrm{MHz})$ is suppressed. Standard B-Mode, SH filtered and PI approaches are compared on a carotid artery phantom in Fig. 2(g-i), demonstrating PI as a superior technique with almost complete suppression of the tissue and much improved contrast agent-to-tissue ratio.

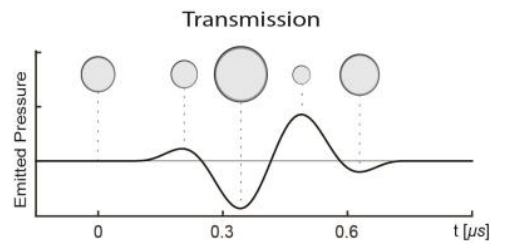

(a) Transmitted US pulse and MB behaviour

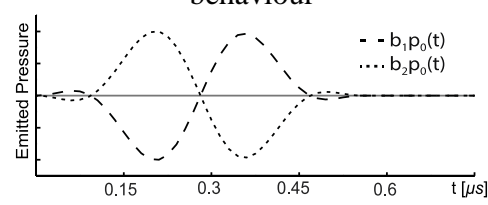

(d) PI transmissions

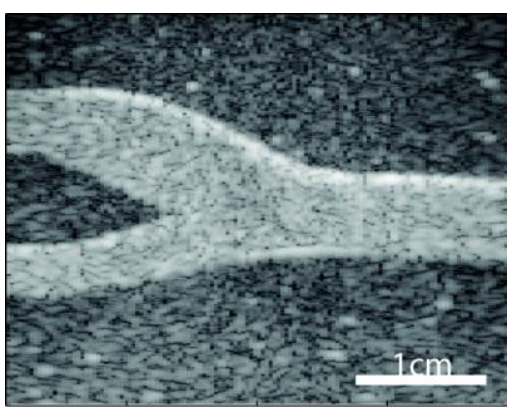

(g) Conventional B-mode image

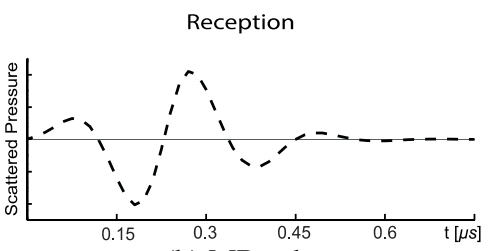

(b) MB echo

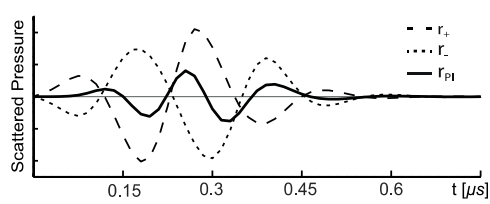

(e) MB echoes before and after PI cancellation

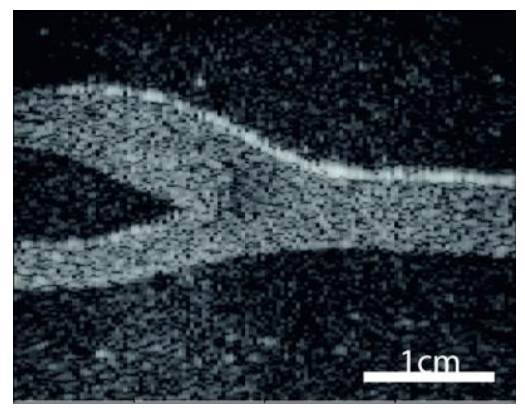

(h) Second harmonic filtered
Power Spectra

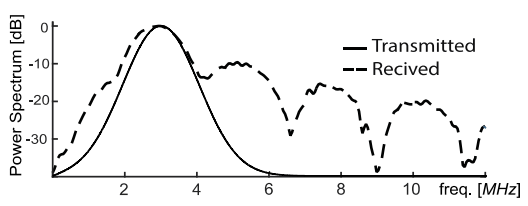

(c) Transmission and MB echo spectra

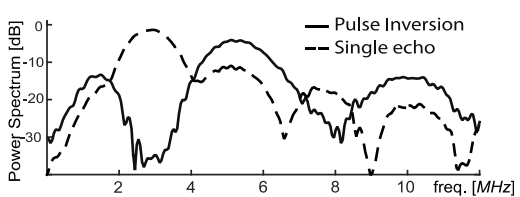

(f) PI spectra before and after cancellation

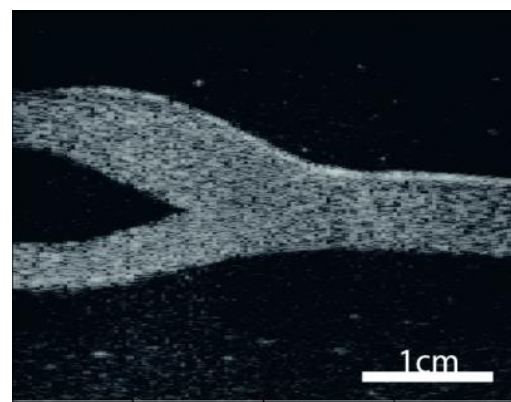

(i) Pulse Inversion

Figure 2: Principles of MB detection. The first row shows (a) microbubble oscillation in response to an external US pressure field, the scattered ultrasound wave (b) and (c) the frequency response. The second row illustrates the principle of Pulse-Inversion detection, where (d) depicts positive and negative transmitted pulses and (e) shows the MB responses to them; the sum of the two responses is shown by the solid line and the frequency spectrum in (f). The last row shows that the Pulse Inversion image (i) has better specificity in imaging microbubbles than 
(g) B-mode and (h) second harmonic filtered images.

There are a number of multi-pulse techniques related to PI which employ varying pulse phase or amplitude. These can be summarised through a mathematical generalization [4]. If the transmitted US wave is given by:

$$
\begin{gathered}
b_{k} p(t)=b_{k} P_{0} e^{j \omega_{0} t+\varphi_{0}} \\
b_{k}=\left|b_{k}\right| e^{j \varphi_{k}}, \quad \varphi_{k} \in[0,2 \pi], k=1,2, \ldots, K
\end{gathered}
$$

where $p(t)$ represents the basic pulse, $P_{0}$ the amplitude of the wave, $\omega_{0}$ the US angular frequency and $\varphi_{0}$ the phase. $b_{k}$ indicates the changes in amplitude and phase applied to the $k$-th transmitted pulse. The $k$-th received wave can then be modelled as a Taylor series:

$$
r_{k}(t)=\sum_{n=1}^{N} a_{n}\left[b_{k} p(t)\right]^{n}
$$

where $a_{n}$ is the weight of the $n$-th nonlinear component and $N$ is the model order. Given a sinusoidal $p(t)$, the $n$-th nonlinear component contains the $n$-th harmonic. After consecutive transmission and reception of $K$ pulses in the medium, the post-processing to generate contrast specific signals can be done by a weighted sum of the received pulses:

$$
r_{\text {sum }}(t)=\sum_{k=1}^{K} \lambda_{k} r_{k}(t)=\sum_{n=1}^{N}\left(\sum_{k=1}^{K} \lambda_{k} b_{k}^{n}\right) a_{n} p^{n}(t)
$$

where $\lambda_{k}$ is a reception coefficient that must be appropriately chosen depending on $b_{k}$ in order to cancel linear components. The transmission and reception parameters of PI, amplitude modulation (AM) and contrast pulse sequence (CPS) are given in Table 1 [4].

\begin{tabular}{c||c|c|c} 
Pulse-sequence & $b$ & $\lambda$ & $r_{\text {sum }}(t)$ \\
\hline \hline PI & $\left(1, e^{j \pi}\right)$ & $(1,1)$ & $\sum_{n=1}^{N}\left(1+e^{j n \pi}\right) a_{n} p^{n}(t)$ \\
\hline \multirow{2}{*}{ AM } & $\left(1, \frac{1}{2}\right)$ & $(1,-2)$ & $\sum_{n=1}^{N}\left(1-2^{1-n}\right) a_{n} p^{n}(t)$ \\
\hline CPS & $\left(1, \frac{1}{2} e^{j \pi}, \frac{1}{2} e^{j \pi}\right)$ & $(1,1,1)$ & $\sum_{n=1}^{N}\left(1+2^{1-n} e^{j n \pi}\right) a_{n} p^{n}(t)$
\end{tabular}

Table 1. Multi-pulse techniques

While multi-pulse transmission reduces the imaging frame rate, this can be compensated by the emerging ultrafast imaging techniques with up to tens of thousands of frames per second $[5]$.

\subsection{Encoded Pulses}

Even at moderate US amplitudes (tens to hundreds of kilo-Pascals), MBs can be disrupted. 
This occurs because the negative pressure portion in the US pulse can cause the shell to expand until it ruptures. To mitigate this effect, the transmit amplitude needs to be kept low, with a consequent reduction in the signal-to-noise ratio (SNR). Transmitting longer pulses can increase the signal energy and consequently the SNR, without increasing the peak pressures and the associated risk of $\mathrm{MB}$ destruction, but this comes at the cost of a reduced image resolution. Coded transmission techniques, such as frequency encoding (chirp), were developed for radar systems for the conflicting requirements of simultaneous high SNR and high-resolution performance. A long pulse with an embedded code is transmitted. After reception, the signal is cross-correlated with the transmitted pulse to detect and remove the code and restore the spatial resolution in a process called compression.

A nonlinear compression filter can be used to selectively extract and compress the SH from the received echo of MBs [6]. While this combination of harmonic imaging and chirp transmission improves the resolution of the technique, side lobes can appear due to the chirp compression and frequency overlapping. The method can be improved by extracting the chirp SH component in a space between the time and frequency using the fractional Fourier transform (FrFT) [7]. Furthermore, pulse encoding is not limited to frequency encoding (chirp) and other codes such as Barker and Golay codes can also be used.

\section{Detecting bubble motion using non-linear Doppler processing}

The measurement of MB motion can provide insight about the flow conditions (fast vs. slow), and is useful for determining whether targetted bubbles are bound or free flowing. Multi-pulse sequences can be used to both detect the existence of MBs as described above and to provide motion information. The Doppler equation describes the frequency shift given by the relative motion between a sound source and an observer. In US imaging, it can be used to determine movement of objects such as red blood cells or MBs. For the sake of simplicity, let us focus on motion along the US propagation direction only. The frequency shift of a linear scatter moving with velocity $v$ is given by:

$$
\Delta f=\frac{2 f_{0} v}{c}
$$

where $f_{0}$ is the fundamental frequency of the transmitted pulse and $c$ the speed of sound. Continous wave Doppler has great velocity resolution but does not offer spatial information. This can be overcome by sending multiple pulses at a frequency $f_{\mathrm{PRF}}$ and measuring the relative phase shift of the responses over time $\Delta \phi(t)$, a technique known as Pulsed-Doppler. The Fourier transform of $\Delta \phi(t)$ will return the frequency shift, provided that the motion is slow 
enough to be captured at the given $f_{\mathrm{PRF}}$ (Nyquist constraint).

A moving non-linear scatter like microbubbles will generate both fundamental and harmonic Doppler signals of different phase shifts (e.g. $2^{\text {nd }}$ harmonics have phase shifts that are double that of fundamental signals). This has been used in conjunction with PI to distinguish the movement of MB from tissue [2]. As in PI, the pulses used to generate the Doppler signal $\Delta \phi(t)$ are sent with a phase shift of $\pi$ between consecutive pulses, so that the phase shift in the consecutive echoes from a moving linear scatterer has an additional $\pi$ :

$$
\Delta \phi=2 \pi f_{0} \Delta \tau+\pi
$$

where $\Delta \tau=2 v / c f_{\mathrm{PRF}}$ is the time shift due to target motion. The additional $\pi$ due to pulse inversion will shift the spectrum of the Doppler signal $\Delta \phi(t)$ for a linear target by a factor of $1 / 2 f_{\mathrm{PRF}}$. On the other hand, it does not affect the even harmonics emitted by a non-linear target, as can be seen through decomposing the nonlinear signals as in Eq. (3). The Doppler frequency for the $n$-th harmonic can thus be expressed as:

$$
\begin{aligned}
& f_{\mathrm{d}}=\frac{2 f_{0} v}{c} \text { if } n \text { is even } \\
& f_{\mathrm{d}}=\frac{2 f_{0} v}{c}+\frac{1}{2} f_{\mathrm{PRF}} \text { if } n \text { is odd. }
\end{aligned}
$$

Using a low-pass filter with cut-off frequency at $1 / 4 f_{\mathrm{PRF}}$, it is possible to distinguish between even harmonics emitted by MBs and fundamental signals emitted by tissue, even if they have similar motion. This enables the specific imaging of both fast and slowly moving agents in macro- and micro-vessels (see Fig. 1(d)) or potentially those targeted agents adherent to a vessel.

\section{Artefact Reduction for Contrast Detection}

CEUS suffers from various imaging artefacts [8] and signal processing algorithms have been developed to cope with them. One type of common artefact arises from the nonlinear distortion of the transmitted US pulse as it propagates through tissue and MB clouds, generating harmonics. The degree of the nonlinear distortion depends on both the US amplitude and the medium. These propagation harmonics are subsequently reflected by tissues and then misclassified as MB. This is commonly referred to as a nonlinear propagation artefact and can be observed below the vessel wall in Fig. 3(a).

Among the different techniques developed to mitigate such artefacts, the method in [9] uses a signal processing approach where the nonlinear tissue artefact is estimated based on two simultaneously acquired images: 1) the B-mode image that contains mainly tissue signals, and 2) the contrast specific image (such as PI) that contains both MB signals and artefacts. As the 
artefacts originate from tissue reflection/scattering, removing artefacts means estimating and removing the tissue signals (found in the B-mode image) from the contrast specific image. As the B-mode and the contrast specific images have different point spread functions (PSFs), direct subtraction does not work. In [9], the tissue scatterer distribution (TSD) is first estimated by de-convolving the B-mode image with the measured linear PSF of the system and the artefact is then estimated by convolving the TSD with the measured nonlinear PSFs. Fig. 3(a-b) shows the original and corrected PI images in a flow phantom (i.e. an artificial model of human vessels embedded in tissue). MB signal from a small vessel (indicated by arrows in Fig 3.(a-b)) buried within tissue artefacts prior to correction has been revealed.

Another common image artefact is due to the attenuation of the ultrasound [8], especially from the probe-skin interface and patient-specific, spatially heterogeneous, attenuation which often introduces a vertical shadow in the image (Fig. 3(c) arrow). Correcting this attenuation is crucial for blood flow quantification using CEUS. In [10], an algorithm has been developed to correct for attenuation artefacts with the prior knowledge that MBs are well mixed and homogeneous throughout the interior of a vessel. The relative attenuation profile in the vessel is estimated by lowpass filtering the image. Then the measured attenuation profile is expanded to adjacent regions of interest such as vessel walls, Fig. 3(c-d).

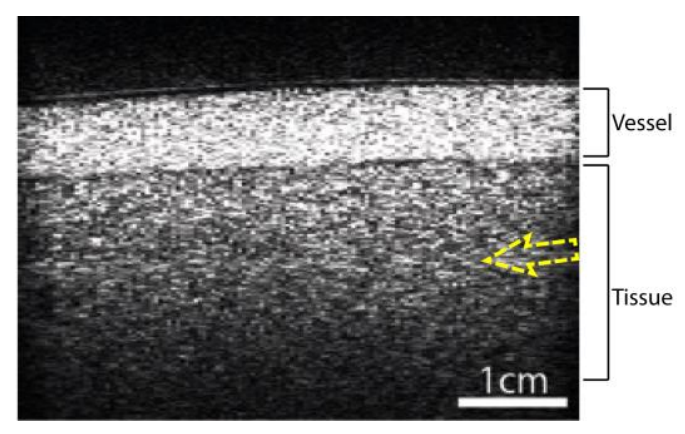

(a) Pulse Inversion

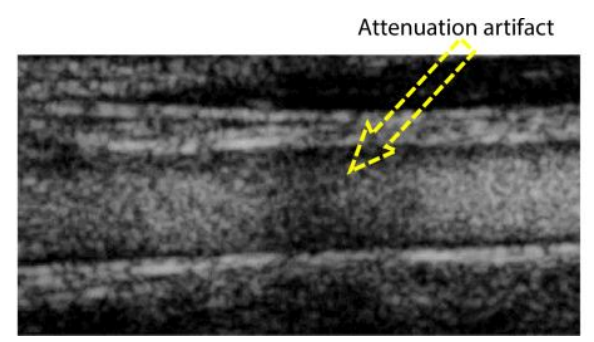

(c) Ultrasound attenuation artefact

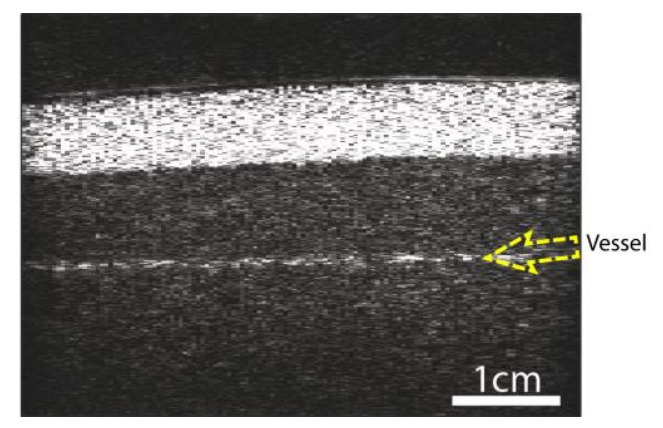

(b) Corrected Pulse Inversion

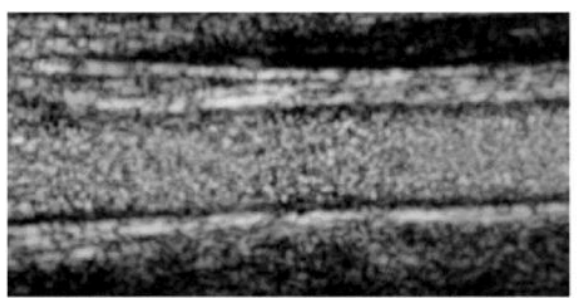

(d) Corrected attenuation artefact

Figure 3: Imaging artefact correction: (a) Pulse inversion and (b) corrected Pulse Inversion images of a flow phantom with two vessels containing diluted suspension of MBs (adapted from [9]), where the yellow arrows point to the small vessel recovered by the artefact correction. In-vivo CEUS image of a carotid artery with (c) attenuation artefact (yellow arrow) 
and (d) attenuation artefact corrected (adapted from [10]).

\section{Image Processing and Analysis}

CEUS data encompasses both spatial and temporal information. MB signals in US image sequences can indeed be analysed over space and time to derive valuable information e.g. arterial blood flow fields, micro-vascular morphology, or tissue perfusion indices, in cardiovascular diseases and cancer for instance.

Perfusion Quantification: A unique feature of MBs as contrast agents is that they can be deactivated (destroyed) by using high amplitude (but still safe) US pulses. It enables a well-controlled local input function (step function), and offers means to study local micro-flow dynamics through a technique referred to as destruction reperfusion analysis [11]. A high amplitude US destruction pulse is transmitted to wipe out MBs within a region of tissue and subsequent low amplitude US imaging pulses are then used to observe the replenishment of MB signals. Examples of myocardium reperfusion are shown in Fig. 4(a), where the top segment of the myocardium (red line) shows much lower perfusion than the other segment (blue line), indicating a possible stenosis in the coronary artery. Such reperfusion curves (Fig. 4(a)) can be fitted to a perfusion model, e.g. $I=A\left(1-e^{-\beta t}\right)$ where physiologically relevant quantities such as blood volume $(A)$ and flow rate $(\beta)$ can be extracted.

Super-Resolution Imaging: MBs provide an opportunity to reveal extra structural information of the vasculature with far more details than what the diffraction limit of US might suggest. As presented above, the non-linear properties of the MBs facilitate their detection with very high sensitivity, so that an individual MB can scatter signals far above the noise floor of the US system. Borrowing ideas from similar approaches in optical super-resolution microscopy, it has been shown that by localising the detected 2D signals from individual $\mathrm{MB}$, using simple spatial filtering tools, vessels $<20 \mu \mathrm{m}$ can be delineated, see Fig. $4(\mathrm{~b})$. This is much smaller than the diffraction limited resolution of $\sim 100 \mu \mathrm{m}$ in this example [12]. 

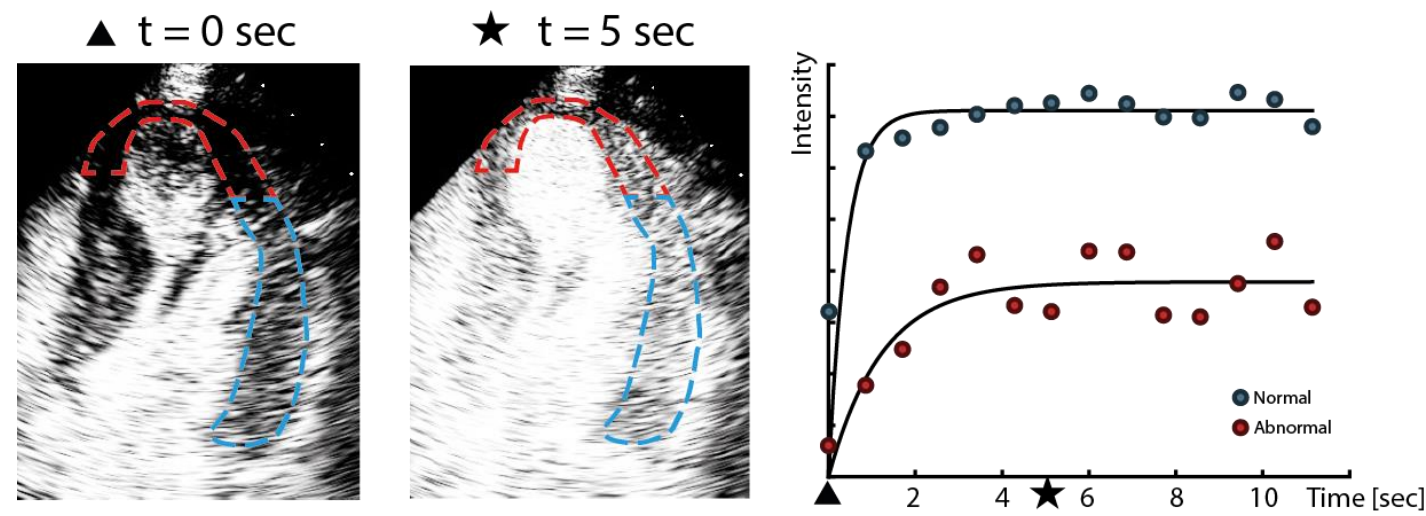

(a) Heart perfusion

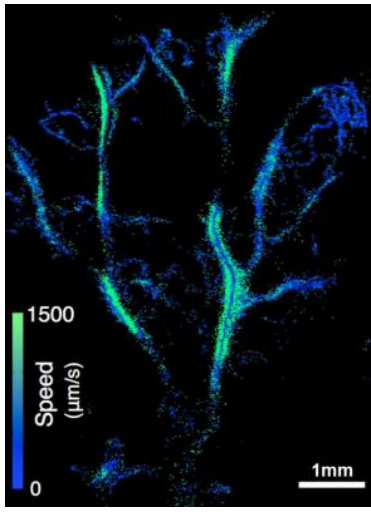

(b) Super resolution

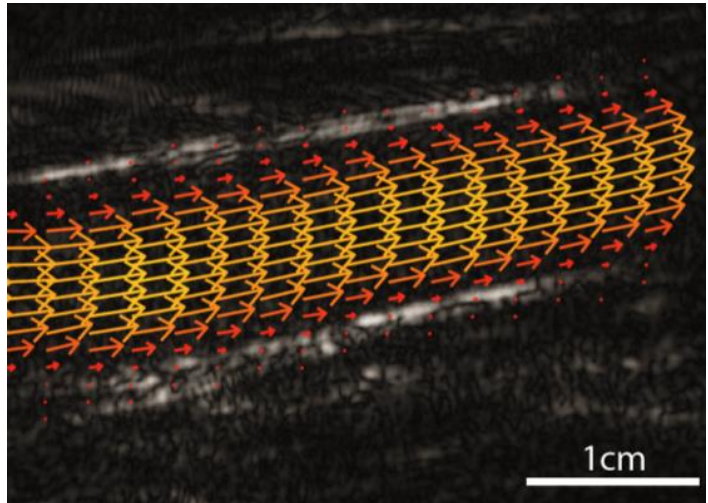

(c) Ultrasound imaging velocimetry

Figure 4: Applications of contrast enhanced ultrasound imaging: (a) left ventricle myocardium perfusion shown by CEUS at two time points and its quantification, (b) super resolution ultrasound imaging (reproduced from [12]) in vivo and (c) blood flow velocity estimation using MB tracking in a rabbit abdominal aorta (adapted from [13]).

Bubble Tracking: Temporal tracking of MBs offers new opportunities in terms of mapping blood flow and vascular morphology. Combined with very high frame-rate ultrasound currently available (up to 20k frames per second), it has been used for mapping rapid arterial flow in Ultrasound Imaging Velocimetry (UIV) (also called echo Particle Image Velocimetry, e-PIV) [13], see Fig. 4(c). Furthermore, tracking individual MBs in perfused tissue can help segment small vessels in the CEUS images and offers a valuable measure of vascular density.

In the reported MB tracking techniques, it is common practice to use cross-correlation. Depending on the prior knowledge of the flow, some applications require an iterative process to refine the size of the spatial window in which the cross-correlation is calculated. There is a significant amount of literature in signal processing on target tracking which could potentially benefit this area and provide more robust and real-time tracking of flow dynamics. 


\section{Final Remarks}

US imaging with MBs offers exciting opportunities for revealing the structure and function of the vascular network with unprecedented sensitivity and resolution. The on-going demand for more specific detection of MBs, together with the rich data generated in both space and time by CEUS, presents significant opportunities and challenges for the development of signal processing tools and solutions. A notable recent development in US imaging is the capability of imaging at a frame rate of up to tens of thousands frames per second [5]. While such ultrafast imaging technology offers great opportunities for the improvement of US imaging, especially for fast moving objects such as a beating heart, arterial flow, or a shear wave within the tissue based on which the tissue elasticity can be quantified, it also raises significant challenges. Techniques to make full use of the GB of data acquired per second are required. There are opportunities to take advantage of the prior knowledge in both the underlying imaging physics and target tissue/organ physiology, and to generate in real-time clinically relevant information which are yet to be fully exploited. In addition, the complex nonlinear signals generated by MBs provide another avenue for research, as the MB signals can be influenced by many variables related to the in-vivo environment, such as blood pressure, proximity to vessel wall, gas saturation, and the mechanical properties of the surrounding tissue. A better understanding of the physics and advanced modelling and signal processing techniques could lead to extracting this clinically relevant information from the MB signals. Additionally, while most clinical US imaging is still in two dimensions, three-dimensional US imaging is arriving and will create further opportunities and challenges for data post-processing. Finally, molecular imaging using targeted MBs is another exciting area of further development, where more advanced signal processing could help detect and evaluate pathologies at their earliest stage.

\section{Acknowledgments}

The authors would like to thanks Dr. Adrian Lim and Professor David Cosgrove for providing some of the clinical images, Dr Alfred Yu for providing the flow phantom, and UK EPSRC for the financial support (EP/M011933/1).

\section{Authors}

Antonio Stanziola (antonio.stanziola14@ic.ac.uk) is a PhD student at the Ultrasound Laboratory for Imaging and Sensing (ULIS) group, Imperial College London. Matthieu Toulemonde (m.toulemonde@ic.ac.uk) is a postdoc researcher at the Ultrasound Laboratory for Imaging and 
Sensing (ULIS) group, Imperial College London. Yesna O. Yildiz (y.yildiz11@ic.ac.uk) is a $\mathrm{PhD}$ student at the Ultrasound Laboratory for Imaging and Sensing (ULIS) group, Imperial

College London. Robert Eckersley (robert.eckersley@kcl.ac.uk) is a Senior Lecturer at King's College London.

Meng-Xing Tang* (mengxing.tang@ic.ac.uk) is a Reader (Associate Professor) in Biomedical Imaging and the head of the Ultrasound Laboratory for Imaging and Sensing (ULIS) group,

Department of Bioengineering, Imperial College London.

\section{References}

[1] J. R. Lindner, "Microbubbles in medical imaging: current applications and future directions", Nat Rev Drug Discov, vol. 3, no. 6, pp. 527-533, 2004.

[2] C. Tremblay-Darveau, R. Williams, L. Milot, M. Bruce, and P.N. Burns, "Combined perfusion and Doppler imaging using plane-wave nonlinear detection and microbubble contrast agents", IEEE Transactions on Ultrasonics, Ferroelectrics, and Frequency Control, vol. 61, no. 12, pp. 1988-2000, 2014.

[3] T. Faez, M. Emmer, K. Kooiman, M. Versluis, A.F.W. van der Steen, and N. de Jong, "20 years of ultrasound contrast agent modeling", IEEE Transactions on Ultrasonics, Ferroelectrics, and Frequency Control, vol. 60, no. 1, pp. 7-20, 2013.

[4] F. Lin, C. Cachard, F. Varray, and O. Basset, "Generalization of multipulse transmission techniques for ultrasound imaging", Ultrasonic Imaging, vol. 37, no. 4, pp. 294-311, 2015.

[5] M. Tanter and M. Fink, "Ultrafast imaging in biomedical ultrasound", IEEE Transactions on Ultrasonics, Ferroelectrics, and Frequency Control, vol. 61, no. 1, pp. 102-119, 2014.

[6] J. M.G. Borsboom, C. T. Chin, A. Bouakaz, M. Versluis, and N. de Jong, "Harmonic chirp imaging method for ultrasound contrast agent", IEEE Transactions on Ultrasonics, Ferroelectrics and Frequency Control, vol. 52, no. 2, pp. 241-249, 2005.

[7] S. Harput, J. McLaughlan, D.M.J. Cowell, and S. Freear, "Superharmonic imaging with chirp coded excitation: filtering spectrally overlapped harmonics", IEEE Transactions on Ultrasonics, Ferroelectrics, and Frequency Control, vol. 61, no. 11, pp. 1802-1814, 2014.

[8] M.-X. Tang, H. Mulvana, T. Gauthier, A. K. P. Lim, D. O. Cosgrove, R. J. Eckersley, and E. Stride, "Quantitative contrast-enhanced ultrasound imaging: a review of sources of variability", Interface Focus, vol. 1, no. 4, pp. 520-539, 2011.

[9] Y. O. Yildiz, R. J. Eckersley, R. Senior, A. K.P. Lim, D. Cosgrove, and M.-X. Tang, "Correction of non-linear propagation artifact in contrast-enhanced ultrasound imaging of carotid arteries: methods and in vitro evaluation", Ultrasound in Medicine \& Biology, vol. 41, no. 7, pp. 1938-1947, 2015.

[10] W. K. Cheung, D. M. Gujral, B. N. Shah, N. S. Chahal, S. Bhattacharyya, D. O. Cosgrove, R. J. Eckersley, K. J. Harrington, R. Senior, C. M. Nutting, and M.-X. Tang, "Attenuation correction and normalisation for quantification of contrast enhancement in ultrasound images of carotid arteries", Ultrasound in Medicine \& Biology, vol. 41, no. 7, pp. 1876-1883, 2015.

[11] K. Wei, A. R. Jayaweera, S. Firoozan, A. Linka, D. M. Skyba, and S. Kaul, "Quantification of myocardial blood flow with ultrasound-induced destruction of microbubbles administered as a constant venous infusion", Circulation, vol. 97, no. 5, pp. 473-483, 1998.

[12] K. Christensen-Jeffries, R.J. Browning, Meng-Xing Tang, C. Dunsby, and R.J. Eckersley, 
"In vivo acoustic super-resolution and super-resolved velocity mapping using microbubbles", IEEE Transactions on Medical Imaging, vol. 34, no. 2, pp. 433-440, 2015.

[13] C. H. Leow, E. Bazigou, R. J. Eckersley, A. C.H. Yu, P. D. Weinberg, and M.-X. Tang, "Flow velocity mapping using contrast enhanced high-frame-rate plane wave ultrasound and image tracking: methods and initial in vitro and in vivo evaluation", Ultrasound in Medicine \& Biology, vol. 41, no. 11, pp. 2913-2925, 2015. 